\title{
Impact of the PPG sampling rate in the pulse rate variability indices evaluating several fiducial points in different pulse waveforms
}

\author{
María Dolores Peláez-Coca, Alberto Hernando, Jesús Lázaro and Eduardo Gil
}

\begin{abstract}
The main aim of this work is to study the effect of the sampling rate of the photoplethysmographic (PPG) signal for pulse rate variability (PRV) analysis in the time and frequency domains, in stationary conditions. Forehead and finger PPG signals were recorded at $1000 \mathrm{~Hz}$ during a rest state, with red and infrared wavelengths, simultaneously with the electrocardiogram (ECG). The PPG sampling rate has been reduced by decimation, obtaining signals at $500 \mathrm{~Hz}, 250 \mathrm{~Hz}, 125 \mathrm{~Hz}, 100 \mathrm{~Hz}, 50 \mathrm{~Hz}$ and 25 Hz. Five fiducial points were computed: apex, upslope, medium, line-medium and medium interpolate point. The medium point is located in the middle of the up-slope of the pulse. The medium interpolate point is a new proposal as fiducial point that consider the abrupt up-slope of the PPG pulse, so it can be recovered by linear interpolation when the sampling rate is reduced. The error performed in the temporal location of the fiducial points was computed. Pulse period time interval series were obtained from all PPG signals and fiducial points, and compared with the RR intervals obtained from the ECG. Heart rate variability and PRV signals were estimated and classical time and frequency domain indices were computed. The results showed that the medium interpolate point of the PPG pulse was the most accurate fiducial point under different PPG morphologies and sensor locations, when sampling rate was reduced. The error in the temporal location points and in the estimation of time and frequency indices was always lower when medium interpolate point was used for all considered sampling rates and for both signals, finger and forehead. The results also showed that the sampling rate of PPG signal can be reduced up to $100 \mathrm{~Hz}$ without causing significant changes in the time and frequency indices, when medium interpolate point was used as fiducial point. Therefore, the use of the medium interpolate point is recommended when working at low sampling rates.
\end{abstract}

Index Terms-Photoplethysmographic, sampling rate, fiducial point, waveform, pulse rate variability.

\section{INTRODUCTION}

Currently a large number of smart wearables are hitting the market. These devices allow the recording of various physiological signals such as electrocardiographic signal (ECG), photopletysmographic signal (PPG) or the blood oxygen saturation, which arouses interest in their possible applications in health, research, sports, etc. Of these signals, the PPG can be registered in various parts of the body with a simple and lowcost technique, by a comfortable small sensor that is based

M.D. Peláez-Coca and A. Hernando are with Centro Universitario de la Defensa (CUD), Zaragoza, Spain.

M.D. Peláez-Coca, A. Hernando, Jesús Lázaro and E. Gil are with BSICoS group, Aragón Institute of Engineering Research (I3A), IIS Aragón, University of Zaragoza, Zaragoza, Spain and E. Gil is with Centro de Investigación Biomédica en Red Bioingeniería, Biomateriales y Nanomedicina (CIBERBBN), Madrid, Spain. on a low-cost technique. The possibility of recording PPG on the wrist by using a sensor integrated in a watch-kind device makes it very interesting for wearable devices. For this reason, the use of this technology is currently spreading remarkably in many different everyday applications, such as sports performance, stress indicator, heart rate control, etc.

PPG obtained can been used to calculate the pulse rate as a surrogate for heart rate, as well as the Pulse Rate Variability signal (PRV) is a surrogate measure for the Heart Rate Variability (HRV) [1]-[3]. The analysis of HRV/PRV is the non-invasive measurement most commonly used to evaluate the activity of the Autonomic Nervous System (ANS) and the balance between its two branches: sympathetic and parasympathetic. The classical analysis of the HRV/PRV [1], [4] considers the power in the low-frequency band (LF, 0.04 $0.15 \mathrm{~Hz})$ as a measurement of both sympathetic and parasympathetic systems, whereas the power in the high-frequency band (HF, $0.15-0.4 \mathrm{~Hz})$ is considered a measurement of parasympathetic activity. The ratio between $\mathrm{LF}$ and $\mathrm{HF}\left(R_{\mathrm{LFHF}}\right)$ is used to estimate the balance between the two branches.

Wearable devices have very limited memory storage, computing power and battery capacity, so it is key to be able to minimize the sampling rate necessary to carry out an optimal PRV analysis, with the least possible consumption of resources. With this in mind, finding the optimal or minimal sampling rate that allows to maintain a correct PPG recording to process an optimal PRV analysis is essential to extend the use of applications based on the PPG signal. In fact, the PPG signal has been used in the study of the ANS response to stress situations, variable hyperbaric environments, or for the diagnosis of obstructive sleep apnea [5]-[8]. The development of applications based on the results of these studies would be expanded if the algorithms could be adapted to a wearable.

Photoplethysmography is a non-invasive optical technique measured by pulse oximeter. This technique is based on the interaction between light and hemoglobin present in the bloodstream during the perfusion of blood in the dermis and subcutaneous tissue. This dermal blood flow is modified by multiple physiological systems at the same time, so PPG can be used to measure heart rate, but also to monitor respiration, hypovolemia and other circulatory phenomena [9]. The measurement of the PPG signal can be performed by transmission or reflection of the light emitted by the sensor. Most commercial pulse oximeters measure in transmission, but the number of devices that measure PPG in reflection is increasing as it can be used on practically any surface of the 
human body and not only on the extremities, facilitating its location. These types of measurements usually present a lower optical signal and higher ambient noise. Moreover, different wavelengths can be used to record the PPG signal and each light penetrates human tissue differently, with shorter wavelengths having shorter penetration. Green, red and infrared are the most commonly used wavelengths, being the infrared the one with deepest penetration, and therefore with the possibility of reflecting the blood pulse from deep tissue [10], [11].

Regarding the most appropriate sampling rate, the American Heart Association recommended an ECG sampling rate of $500 \mathrm{~Hz}$ with a resolution of 12 bits [12], although for commercial applications this sampling rate is usually reduced to $128 \mathrm{~Hz}$ [13], [14]. $128 \mathrm{~Hz}$ is a sampling rate with a generally acceptable signal to noise ratio, but it can fluctuate throughout the day. In those cases, the authors propose using techniques such as R-wave interpolation to achieve greater precision in measuring the RR interval [14]. In the case of the PPG signal, it has been studied how a reduction in the sampling rate can affect the analysis of the PRV. In [15] and [16] this reduction in sampling rate was studied in a PPG signal recorded on the finger and using the apex of pulse wave as fiducial point. In [17], the PPG signal was generated on a PC and using the apex of pulse wave as fiducial point. These works did not consider that the shape of the PPG pulse can been smoothed depending on the areas of the body in which it is registered, increasing the error in the location of the apex points when the sampling rate is reduced, or the benefits of using other fiducial points.

However, in the PRV analysis, it is essential to select a fiducial point as consistent as possible, and this results challenging due to the smooth shape of the PPG signal. Different fiducial points have been proposed for the temporal location of each pulse wave in several studies, such as the apex, the up-slope, medium amplitude and foot points of the PPG signal, depending on the application [18]: from the PPG finger for the diagnosis of obstructive sleep apnea [7], [8], from the PPG ear lobe to measure the pulse arrival time [19] or to derive the pulse rate instantly [20].

In this work, a new fiducial point, called medium amplitude interpolate point, is proposed based on the consideration that the up-slope of the PPG pulse is close to be linear, so it can be recovered by linear interpolation when the sampling rate is reduced.

The selection of a consistent fiducial point depend on the morphology of the PPG and the signal acquisition technique [21]. As a sum up, how the reduction of the sampling rate affects the PRV analysis was studied in this work, considering the following variables: 1. two PPG measurement configuration (reflection-based-forehead and transmission-basedfinger); 2. two wavelengths of the PPG optical sensor (red and infrared); 3. five fiducial points (apex, up-slope, medium amplitude, line-medium amplitude and medium amplitude interpolate point).

\section{MATERIALS}

A total of 57 subjects (54 males and 3 females), with a mean age of $30.6 \pm 6.5$ years were recorded in this study. All subjects received the approval of the Comité de ética de la investigación con medicamentos de la inspección general de sanidad de la Defensa (Ethics Committee of Research with Medicines, attached to the Surgeon General of the Undersecretary of Defense), and the participant information sheet and informed consent were signed. During the recordings subjects stayed relaxed and sitting comfortably, remained in silence and without performing movements for five minutes.

The recordings were done using the Nautilus device developed by the University of Kaunas, Lithuania [22]. This device allows to record the PPG signal $\left(f_{s}=1000 \mathrm{~Hz}\right)$ on a finger and forehead with red and infrared wavelengths, and the ECG signal with three leads $\left(f_{s}=2000 \mathrm{~Hz}\right)$. PPG sensors were located on middle finger of the non-dominant hand and on the central area of the forehead fastened with an elastic band.

\section{METHODS}

\section{A. ECG and PPG preprocessing}

In this study, only the last four minutes of the five minutes recorded of the PPG and ECG signals were used, to avoid the possible effect on ANS due to the transition between active and resting subject.

The ECG signal, that will be used as a reference, was first down-sampled to $1000 \mathrm{~Hz}$ to obtain the same sampling rate as the PPG signal. A low-pass FIR filter was then applied to both signals to estimate the baseline interference and to remove it from the signal (cut-off frequency of $0.03 \mathrm{~Hz}$ and $0.07 \mathrm{~Hz}$ for the ECG and PPG signals, respectively) [23]. Heart beats were detected from the frontal bipolar second lead of the recorded ECG signal using an algorithm based on wavelets [24]. Ectopic beats, missed and false detections were identified and corrected [25]. As a result, QRS complex were located in the ECG and the difference between consecutive R waves conformed the RR time series.

In the PPG signal another low-pass FIR filter, with cut-off frequency of $35 \mathrm{~Hz}$, was applied over the PPG signal to remove the high frequency noise [26], obtaining $x_{\mathrm{PPG}}$. Artefactual pulses in the preprocessed PPG signal were suppressed by using the artefact detector described in [27]. Nine subjects were discarded, due to the poor signal-to-noise ratio, for the signal recorded on the forehead. Six new PPG signals were generated reducing the sampling rate by decimation, obtaining: $500 \mathrm{~Hz}, 250 \mathrm{~Hz}, 125 \mathrm{~Hz}, 100 \mathrm{~Hz}, 50 \mathrm{~Hz}$ and $25 \mathrm{~Hz}$. The segments of the PPG signal at $1000 \mathrm{~Hz}$ tagged as artefact by the detector were discarded for the variability analysis in the six decimated PPG signals and in the ECG signal.

\section{B. Fiducial points in the PPG signal}

Five fiducial points have been considered in order to determine if the effect of the sampling rate on time and frequency indices of PRV depends on the selected fiducial point. The apex $\left(n_{\mathrm{Ai}}\right)$ of the $i$-th PPG pulse was automatically detected using an algorithm based on a low-pass differentiator filter and time-varying threshold, that limits the search region of the $n_{\mathrm{Ai}}$ to an interval after the apex of the derivative PPG signal (or up-slope point $n_{\mathrm{Si}}$ ) [7]. $n_{\mathrm{Si}}$ has also been considered as a fiducial point. The medium point $\left(n_{\mathrm{Mi}}\right)$, defined as the 
one in which the amplitude has reached the $50 \%$ of the pulse amplitude. To estimate this point, the basal point $\left(n_{\mathrm{Bi}}\right)$ was defined as the time instant of the minimum pulse amplitude and was calculated as:

$$
n_{\mathrm{Bi}}=\underset{n \in\left[n_{\mathrm{Ai}}-0.3 f_{s}, n_{\mathrm{Ai}}\right]}{\arg \min }\left\{x_{\mathrm{PPG}}(n)\right\}
$$

Using $n_{\mathrm{Ai}}$ and $n_{\mathrm{Bi}}$, the point $n_{\mathrm{Mi}}$ was estimated as:

$$
\begin{aligned}
& x_{\mathrm{Mi}}=\frac{x_{\mathrm{PPG}}\left(n_{\mathrm{Ai}}\right)+x_{\mathrm{PPG}}\left(n_{\mathrm{Bi}}\right)}{2} \\
& n_{\mathrm{Mi}}=\underset{n \in\left[n_{\mathrm{Bi}}, n_{\mathrm{Ai}}\right]}{\arg \min }\left\{\left|x_{\mathrm{PPG}}(n)-x_{\mathrm{Mi}}\right|\right\}
\end{aligned}
$$

The interpolated medium point $\left(n_{\mathrm{Ii}}\right)$ was estimated in the same way as the $n_{\mathrm{Mi}}$, but with a previous linear interpolation at $1000 \mathrm{~Hz}$ of the pulse region between $n_{\mathrm{Ai}}$ and $n_{\mathrm{Bi}}$. This interpolation would minimize the error in the location of the medium point, when the sampling rate is reduced. The $1000 \mathrm{~Hz}$ sampling rate was selected because it was the frequency of the reference signals. To estimate the line-medium point $\left(n_{\mathrm{Li}}\right)$, the equation of the line that passes through the two samples of the PPG pulse closest to the amplitude $x_{\mathrm{Mi}}$ was calculated. Finally, the point $n_{\mathrm{Li}}$ was estimated as the time instant in which said equation takes the value of amplitude $x_{\mathrm{Mi}}$. The $n_{\mathrm{Bi}}$ points was not evaluated in this work, because in previous works it was observed that they were significantly less accurate for PRV analysis than the other considered fiducial points [18].

The difference between consecutive apex $\left(n_{\mathrm{Ai}}\right)$, up-slope $\left(n_{\mathrm{Si}}\right)$, medium $\left(n_{\mathrm{Mi}}\right)$, interpolate medium $\left(n_{\mathrm{Ii}}\right)$ and line-medium $\left(n_{\mathrm{Li}}\right)$ points conforms the pulse period time interval series or PP time series for every sampling rate.

Fig. 1 shows a PPG signal where its more representative points are highlighted.

\section{Time and frequency indices}

Classical time and frequency domain indices from the HRV and PRV signals were computed [4]. Five time indices were computed from the RR and PP time series, and their mean was obtained:

- $\overline{H R M} / \overline{P R M}$ : mean heart/pulse rate, measured in beats per minute (bpm);

- $\overline{S D N N}$ : standard deviation of the Normal-to-Normal (NN) intervals (measure units: ms);

- $\overline{R M S S D}$ : root mean square of the successive differences between adjacent NN intervals. (measure units: ms);

- $\overline{p N N 50}$ : number of pairs of successive NN that differ by more than $50 \mathrm{~ms}$, divided by the total number of $\mathrm{NN}$. (measure units: \%).

With the RR and PP time series, the instantaneous heart/pulse rate signal $\left(d_{\mathrm{XR}}(n)\right.$, where $X$ can be $H$ or $P$, referring to the HRV or PRV estimation, respectively) was obtained at $4 \mathrm{~Hz}$ using cubic spline interpolation. The analysis of the ANS was based on the integral pulse frequency modulation model [28]. Then, the signal $d_{\mathrm{XRM}}(n)$ was defined as an estimation of the time-varying mean heart/pulse rate by low-pass filtering the $d_{\mathrm{XR}}(n)$ signal with a cutoff frequency of $0.03 \mathrm{~Hz}$. The variability signals were defined as the difference:

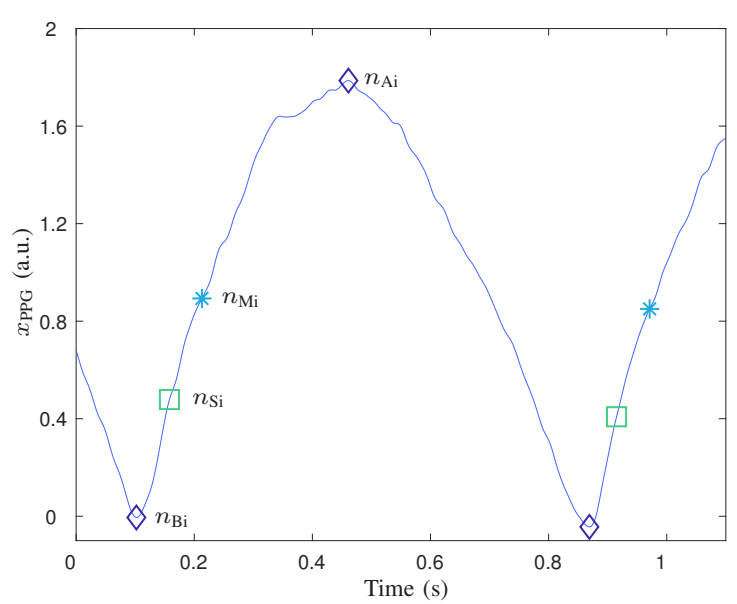

(a) Forehead

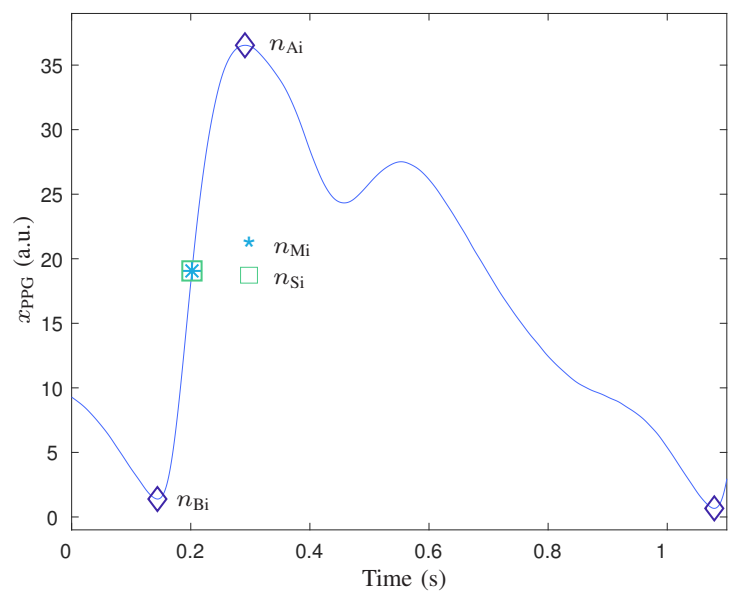

(b) Finger

Fig. 1: Location of the $n_{\mathrm{A}}, n_{\mathrm{B}}, n_{\mathrm{M}}$ and $n_{\mathrm{S}}$ points in the PPG signals at $1000 \mathrm{~Hz}$, recorded on the forehead (top) and the finger (bottom).

$$
d_{\mathrm{XRV}}(n)=d_{\mathrm{XR}}(n)-d_{\mathrm{XRM}}(n)
$$

Finally, the modulating signal, $\mathfrak{M}(n)$, was obtained by correcting $d_{\mathrm{XRV}}(n)$ by $d_{\mathrm{XRM}}(n)$ :

$$
\mathfrak{M}_{X}(n)=\frac{d_{\mathrm{XRV}}(n)}{d_{\mathrm{XRM}}(n)}
$$

Four frequency indices were calculated based on the power spectral distribution (PSD) analysis of $\mathfrak{M}_{X}(n)$, for HRV and PRV signals. As the 4-min-duration recordings were considered stationary, classic frequency domain indices were computed for the $\mathfrak{M}_{X}(n)$ signal using Welch's power spectral density estimation, with seven 1-min-duration Hamming windows and an overlap of $50 \%$. In summary, the following frequency indices were defined:

- $P_{\mathrm{LF}}$ : power in the LF band $(0.04-0.15 \mathrm{~Hz}$; measure units: arbitrary units, a.u.);

- $P_{\mathrm{HF}}$ : power in the HF band $(0.15-0.4 \mathrm{~Hz}$; measure units: a.u.); 
- $P_{\mathrm{LF}_{\mathrm{n}}}$ : power in the $\mathrm{LF}$ band normalized with respect to the powers in the LF and HF bands (measure units: n.u., normalized units): $P_{\mathrm{LF}_{\mathrm{n}}}=P_{\mathrm{LF}} /\left(P_{\mathrm{LF}}+P_{\mathrm{HF}}\right)$;

- $R_{\mathrm{LF} / \mathrm{HF}}$ : ratio between $\mathrm{LF}$ and $\mathrm{HF}$ power (measure units: n.u.): $R_{\mathrm{LF} / \mathrm{HF}}=P_{\mathrm{LF}} / P_{\mathrm{HF}}$.

\section{Performance measurements}

The Shapiro-Wilk test was used to check normality of the data. When the normal distribution was verified the t-Student paired test was applied, when not the Wilcoxon paired test was applied. In the case of multivariate analysis, the Saphiro-Wilk test did not find is a normal distribution of the data, therefore, a non-parametric approach was used for the statistical analyses. Specifically, Friedman tests were applied.

The absolute error in the temporal location of the fiducial point has been calculated as:

$$
e_{\mathrm{a}, \mathrm{yi}}^{f_{s}}=\left|\frac{n_{\mathrm{yi}}^{f_{s}}}{f_{s}}-\frac{n_{\mathrm{yi}}^{1000}}{1000}\right|,
$$

where y denotes the fiducial point (A, S, M, L, or I), and $f_{s}$ the studied sampling rate. A Wilcoxon right-tail paired test was used to quantify the statistical significance of the $e_{\mathrm{a}, \mathrm{yi}}^{f_{s}}$. A $p-v a l u e<\alpha$ defines significance in value, where the significance level $\alpha$ was set to 0.05 .

The agreement between the RR and the different PP series was assessed using median (bias) and limits of agreement (LOA) [29]. The bias or difference median, the interquartile range (iqr) of the difference between methods and the LOA defined as bias $\pm 1.45 \cdot i q r$ values were computed for each fiducial point and $f_{s} .1 .45$ computes the $95 \%$ non-parametric limits of agreement [30].

The relative error obtained in the estimation of the PRV indices with respect to their corresponding HRV and PRV ${ }^{1000}$ indices was calculated, for each fiducial point and each $f_{s}$ :

$$
e_{\mathrm{r}, \mathrm{REF}, \mathrm{yi}}^{f_{s}}=\frac{z_{\mathrm{PRV}, \mathrm{yi}}^{f_{s}}-z_{\mathrm{REF}, \mathrm{yi}}}{z_{\mathrm{REF}, \mathrm{yi}}} \times 100
$$

where $z_{\mathrm{PRV}, \mathrm{yi}}^{f_{s}}$ denotes the studied PRV index $(\overline{P R M}, \overline{S D N N}$, $\overline{R M S S D}, \overline{p N N 50}, P_{\mathrm{LF}}$, or $\left.P_{\mathrm{HF}}\right)$ computed from the fiducial point $y$ using a sampling rate of $f_{s}$, and $z_{\mathrm{REF}, \mathrm{yi}}$ denotes its corresponding HRV or $\mathrm{PRV}^{1000}$ index. In case of $P_{\mathrm{LF}_{\mathrm{n}}}$ and $R_{\mathrm{LF} / \mathrm{HF}}$, the absolute error $\left(e_{\mathrm{a}, \mathrm{REF}, \mathrm{yi}}^{f_{s}}=z_{\mathrm{PRV}, \mathrm{yi}}^{f_{s}}-z_{\mathrm{REF}, \mathrm{yi}}\right)$ was used instead, as their units are already in relative terms.

The t-Student or Wilcoxon paired-test with Bonferroni correction was used to quantify statistical significance of $e_{\mathrm{r}, \mathrm{REF}, \mathrm{yi}}^{f_{s}}$. The significance level $\alpha$ can be $0.05,0.01$ or 0.001 . This statistical analysis was repeated for each fiducial point and all down sampled rates, using PRV from PPG sampled at $1000 \mathrm{~Hz}$ $\left(\mathrm{PRV}^{1000}\right)$ and HRV as reference.

Finally, a Friedman statistical test with Bonferroni correction was applied to assess the differences between the estimated relative error of the five fiducial points.

\section{RESULTS}

\section{A. Location of fiducial points}

The absolute error in the temporal location of the five fiducial points of the PPG signal, when the sampling rate was reduced, can be seen in Fig 2. It can be observed that the error was lower when $n_{\mathrm{L}}$ and $n_{\mathrm{I}}$ were used as fiducial point, for the six studied $f_{s}$. The numbers under the boxplot are the lowest mean value for which Wilcoxon's right-tailed pair test considers that the null hypothesis is not rejected, that is to say, the data comes from a population with this median value. The significance level $\alpha$ was set to 0.05 . 15746 pulses were analysed in the case of the PPG signal recorded on the finger, and 12357 in forehead. In the rest of the paper, only the results for infrared wavelength are shown, beacuse this signal showed a slightly better signal-to-noise ratio. This better signal-to-noise ratio was established based on the total time of the PPG segments not discarded by the artefact detector. The differences in results, between red and infrared wavelength, are described in the text.
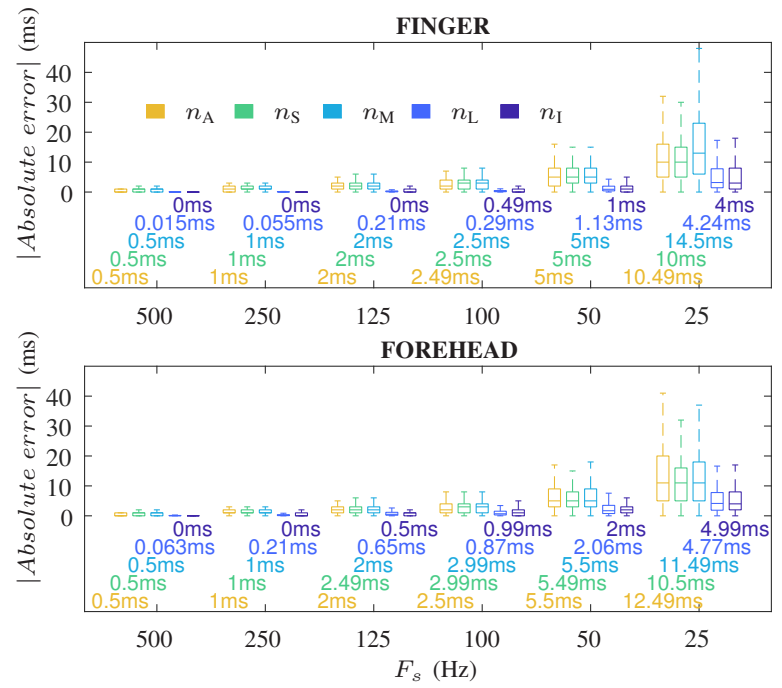

(a) Red
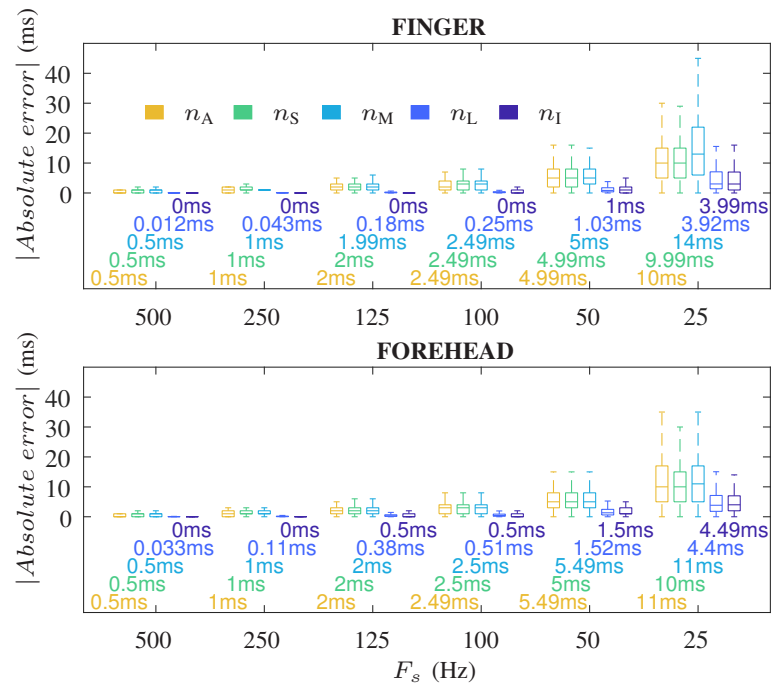

(b) Infrared

Fig. 2: Absolute error obtained in the temporal location of the fiducial point, for a PPG sensor with: (a) red wavelength; (b) infrared wavelength. The numbers in the bottom area of the graph is the maximum median value that rejects the null hypothesis of the Wilcoxon right-tail paired test, for each $f_{s}$ and each fiducial point. The outliers are not plotted. 


\section{B. $R R$ and PP series}

Exact values of bias, iqr and LOA are shown at the Table I. Because of sampling rate of the PPG signal considered, the number of RR and PP paired intervals varied between 14351 and 12161 in the case of the PPG signal recorded on the finger, and between 10410 and 8375 for the one recorded on the forehead. For $n_{\mathrm{M}}, n_{\mathrm{L}}$ and $n_{\mathrm{I}}$ the discrepancies were lower, but in cases where the sampling rate was very low $\left(f_{s}<100 \mathrm{~Hz}\right)$, the best results were obtained with $n_{\mathrm{I}}$. Similar results were obtained for the PPG sensor with red wavelength.

\section{Time and frequency indices}

Table II, Fig. 3 and Fig. 4 show the relative error and absolute error obtained in the estimation of the time and frequency domain indices for the five fiducial points and all sampling rates. Results from finger and forehead PPG were compared to assess the ANS using HRV as reference in Table II, and PRV ${ }^{1000}$ in Fig. 3 and Fig. 4. These results were obtained by averaging among all subjects the indices presented in section III-C, and similar results were obtained for the red wavelength.

The relative error $\left(e_{\mathrm{r}, \mathrm{HRV}, \mathrm{yi}}^{f_{s}}\right)$ in $\overline{P R M}$ and $\overline{S D N N}$ were lower than for the rest of time indices (Table II). In all time and frequency indices a considerably greater error was observed when the fiducial point $n_{\mathrm{A}}$ was used, for the forehead PPG signal, with the exception of $\overline{P R M}$ which is similar for the five fiducial points considered.

Comparing the $e_{\mathrm{r}, \mathrm{PRV}, \mathrm{yi}}^{f_{s}}$ results obtained for each fiducial point (Fig. 3 and Fig. 4), lower relative error in all indices were observed using $n_{\mathrm{I}}$, in finger-PPG. These differences were statistically different from the other fiducial points, according to Friedman statistics with the Bonferroni multiple-comparison test, when the sampling rate decreases, except for the $\overline{P R M}$ and $P_{\mathrm{LF}}$. In forehead-PPG, the best results are obtained with $n_{\mathrm{I}}$ and $n_{\mathrm{L}}$, without significant differences between them, in red and infrared wavelengths. In the case of $n_{\mathrm{I}}$, the median of $e_{\mathrm{r}, \mathrm{PRV}, \mathrm{yi}}^{f_{s}}$ only exceeds $5 \%$ in $\overline{R M S D D}$ and $\overline{p N N 50}$, when the frequency is reduced to $25 \mathrm{~Hz}$.

\section{DISCUSSION}

The main aim of this work was to study the effect of the PPG sampling rate in the analysis of the PRV in stationary conditions, using the HRV and the PRV ${ }^{1000}$ as reference. Five different fiducial points were considered and their suitability for PRV analysis has been studied taking into account the effect of the morphology of the PPG signal, depending on the location and measurement technique of the PPG sensor (finger/transmission, and forehead/reflection). Although some studies suggest than the apex point is not a reliable fiducial point [18], it is still widely used in PRV analysis. The up-slope and the medium point were the two fiducial points that showed the best results in [18]. The line-medium and the medium interpolate point are the two approaches for estimating the medium point when the sample rate is reduced. These approaches are based on assuming that the up-slope of the PPG pulse shows a highly linear slope. This linear interpolation allows the original slope to be accurately estimated, reducing the impact of time resolution (due to sampling) in the location of the medium amplitude point. The method used to locate the line-medium points is equivalent to a linear interpolation with the interpolating sampling rate tending to infinite, but computationally simpler to implement.

In this work, six sampling rates have been considered, which are $1000 \mathrm{~Hz}$ dividers. $500 \mathrm{~Hz}$ and $250 \mathrm{~Hz}$ are sampling rates commonly used in PRV analysis. $200 \mathrm{~Hz}$ has been omitted as it is very close to $250 \mathrm{~Hz}$ and thus reduces the volume of data on the paper. $125,100,50$ and $25 \mathrm{~Hz}$ has been considered to possibly cover the sampling rate of commercial smart bands and watches. The error obtained for $25 \mathrm{~Hz}$ was higher than $5 \%$ in any of the indexes studied (see Fig. 3 and 4), so sampling rates below this value were not considered.

Additionally, the sensor location modifies the morphology of the PPG pulse. In order to generalize our results, two extreme types of PPG morphologies have been considered based on where the analyzed fiducial points are located on the up-slope of the PPG pulse and at its maximum. The shape of this pulse zone when the signal is registered in the finger (in transmission) has a very steep slope and a narrow maximum, while in the forehead (in reflection) the pulse is much more rounded and with a much less steep slope. (Fig. 1). In the case of smart watches, the registration of the PPG signal is usually done on the wrist. The pulse waveforms recorded on the wrist depend on the subject, but would be contained between the two extreme cases studied in this paper [19].

In this work, the PPG signal recordings have been performed using two different wavelengths: $660 \mathrm{~nm}$ for red light and $900-940 \mathrm{~nm}$ for infrared lights. Results showed that the errors in $e_{\mathrm{r}, \mathrm{HRV}, \mathrm{yi}}^{f_{s}}$ were slightly lower when infrared wavelength was used. The used wavelength affects the quality of the PPG signal in several ways. On the one hand, the absorption coefficient of the infrared increases with the hemoglobin oxygenation, being higher than for red [31]. This could increase the dynamic range of the PPG infrared signal, which could reduce the error in location of fiducial points, so the infrared signal may be more suitable for recording the PPG than the red signal. On the other hand, shorter wavelengths have shorter penetration. Therefore, PPG using shorter wavelength optical signals is less influenced by the ambient light (which can be scattered from the close areas), and the movements of the deeper tissues. However, light of shorter wavelengths is strongly absorbed by melanin, so the optimal choice of wavelength depends on the type of the skin of the subject [10], [31]. Infrared PPG showed a better signal-to-noise ratio than red PPG in the studied data. This observation is coherent with [32], where a higher signal-to-noise ratio of infrared PPG was reported.

Results in Fig. 2 show that the error in the location of the fiducial points increases at low sampling rates. However, the smallest error was obtained for $n_{\mathrm{L}}$ and $n_{\mathrm{I}}$ in all the studied cases. Probably, $n_{\mathrm{A}}$ is the most common fiducial point used with a sampling rate of up to $250 \mathrm{~Hz}$. The results seem to indicate that the sampling rate could be reduced to $100 \mathrm{~Hz}$, obtaining similar precisions in the location of the fiducial point, if $n_{\mathrm{L}}$ or $n_{\mathrm{I}}$ is used instead of $n_{\mathrm{A}}$. The small increase in $n_{\mathrm{L}}$ error with respect to $n_{\mathrm{I}}$, for the highest frequencies, is due 

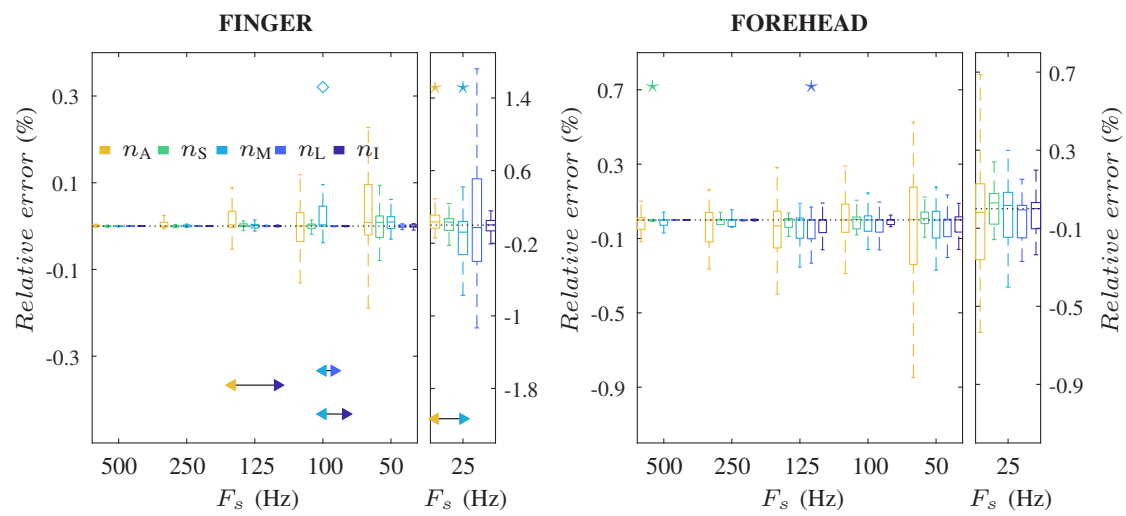

(a) $\overline{P R M}$ : mean pulse rate
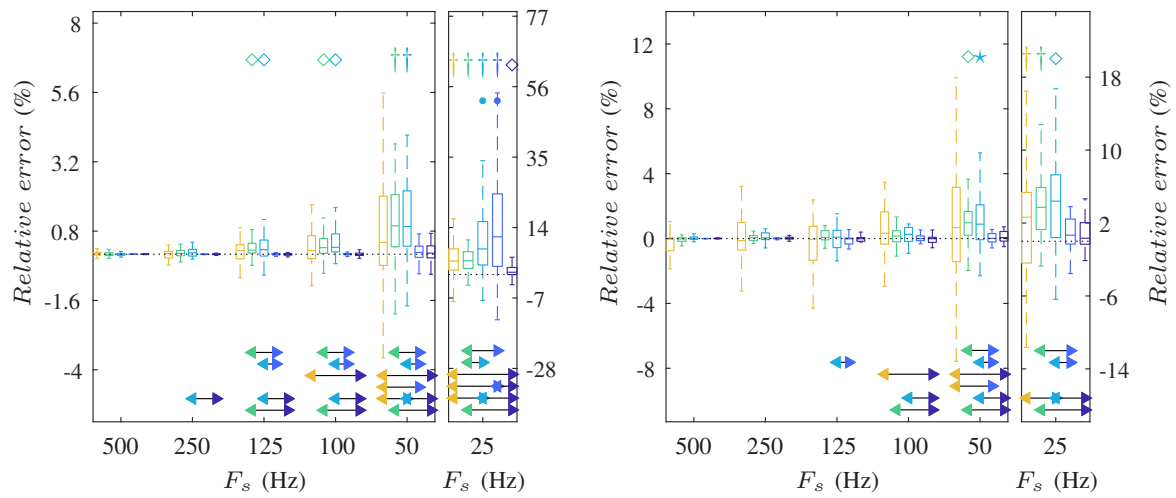

(b) $\overline{S D N N}$ : standard deviation of the NN intervals
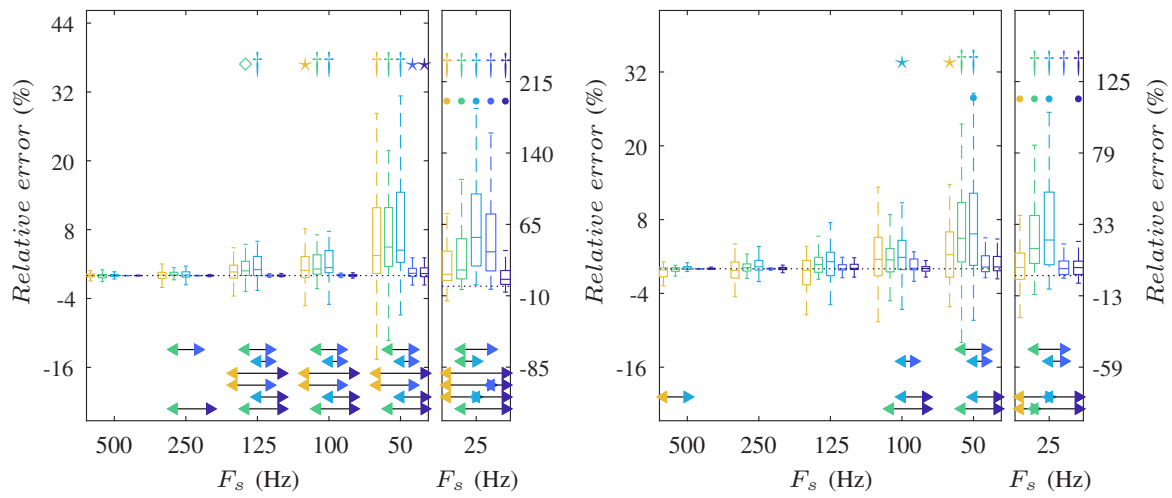

(c) $\overline{R M S S D}$ : root mean square of the successive differences between adjacent NN intervals

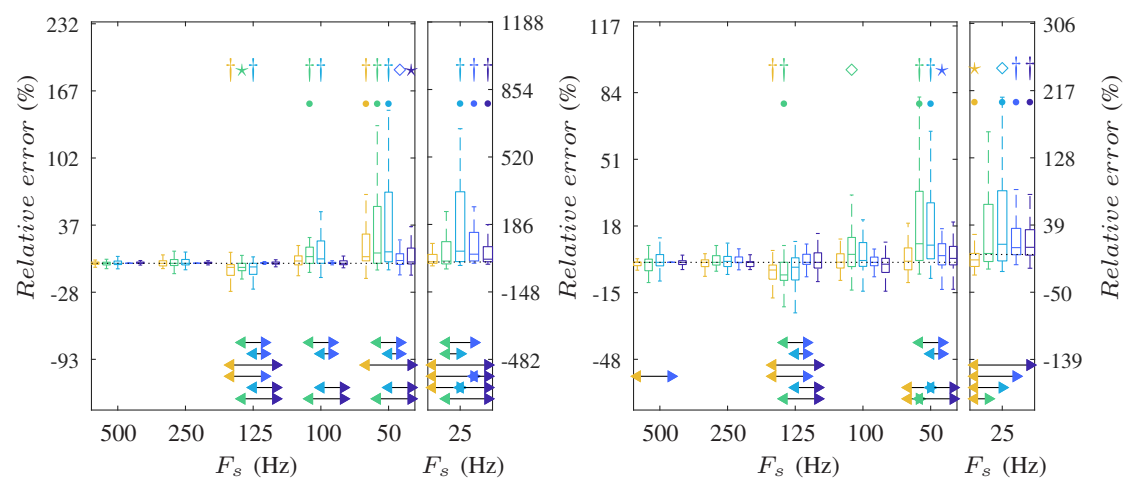

(d) $\overline{p N N 50}$ : percentage of pairs of successive $\mathrm{NN}$ that differ by more than $50 \mathrm{~ms}$

Fig. 3: Estimated relative errors between time indices from PRV and PRV ${ }^{1000}$. The significance level $\alpha$ is indicated with $\star$ for $\alpha=0.05, \diamond$ for $\alpha=0.01$ and $\dagger$ for $\alpha=0.001$. Median of $e_{\mathrm{r}, \mathrm{PRV}, \mathrm{yi}}^{f_{s}}>5 \%$ are indicated by a dot below the $\alpha$ level symbol. The arrow indicates statistically significant differences between fiducial points at the same rate using Friedman statistics with the Bonferroni multiple-comparison test. The colour of the start and end of the arrow indicates the fiducial point analysed. The dotted horizontal line represents the zero value in the error. The outliers are not plotted. 
FINGER

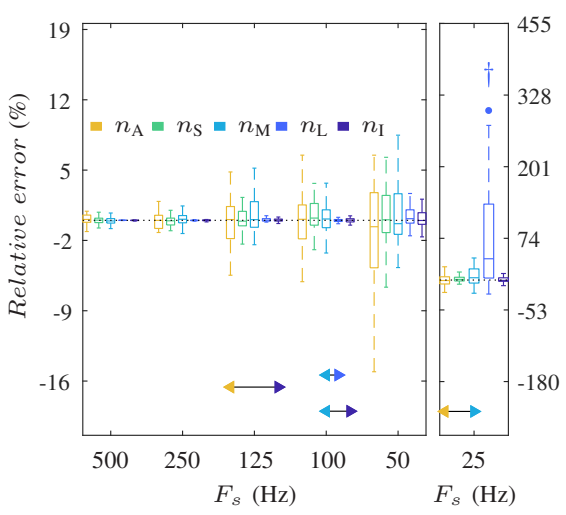

FOREHEAD

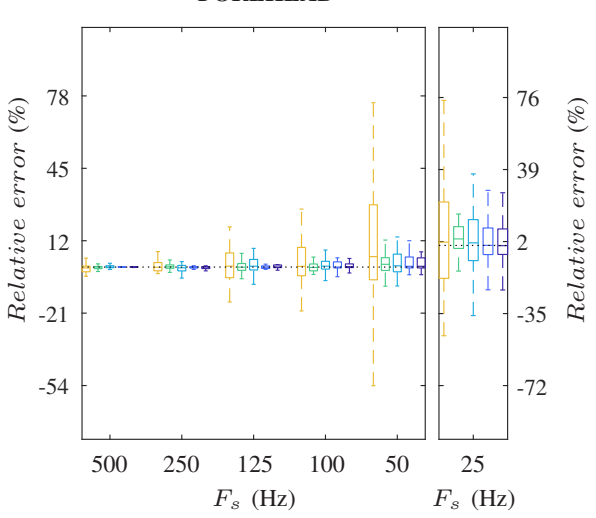

(a) $P_{\mathrm{LF}}$ : power in the LF band $(0.04-0.15 \mathrm{~Hz})$
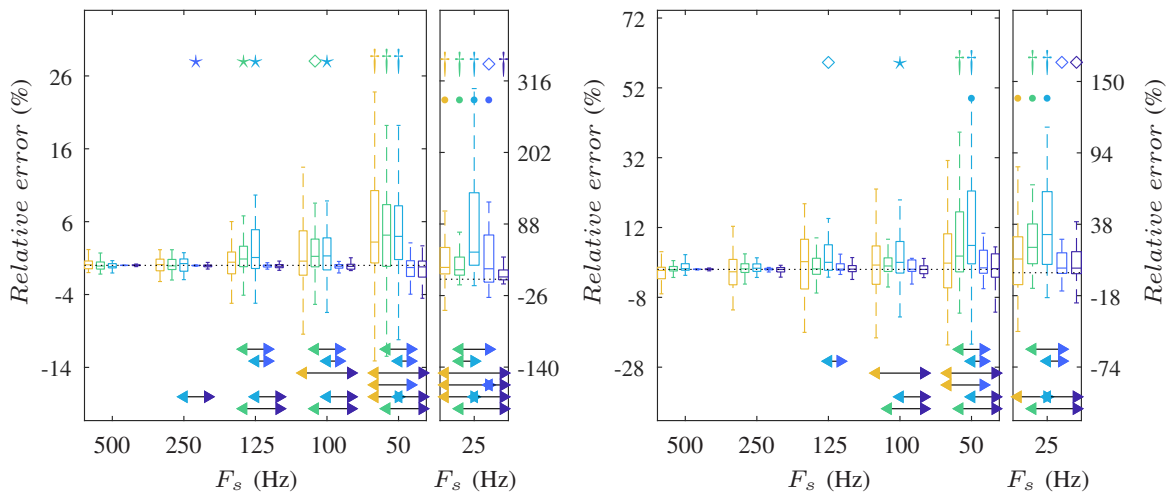

(b) $P_{\mathrm{HF}}$ : power in the $\mathrm{HF}$ band $(0.15-0.4 \mathrm{~Hz})$

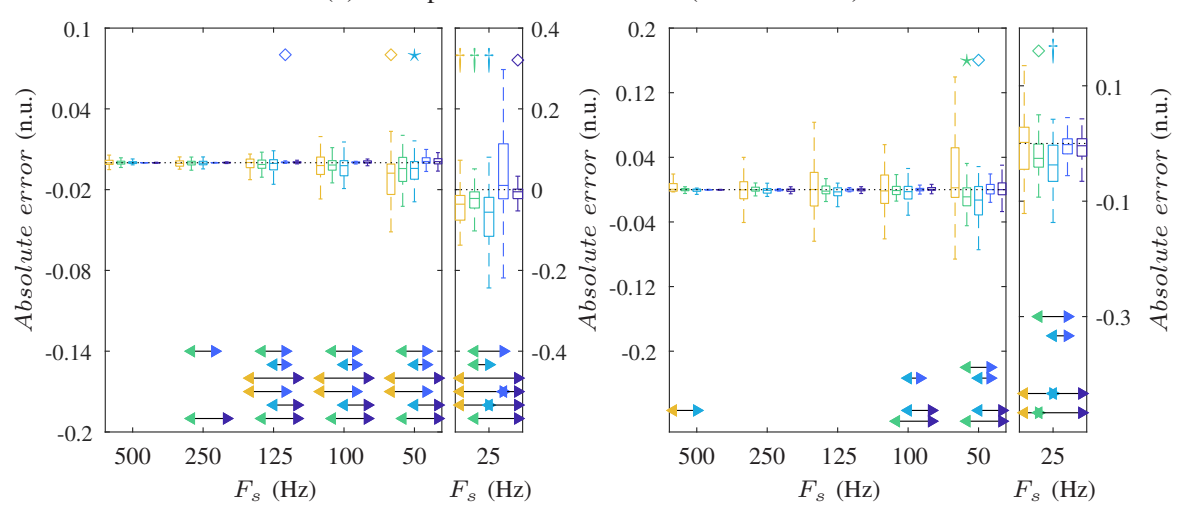

(c) $P_{\mathrm{LFn}}$ : power in the LF band normalized
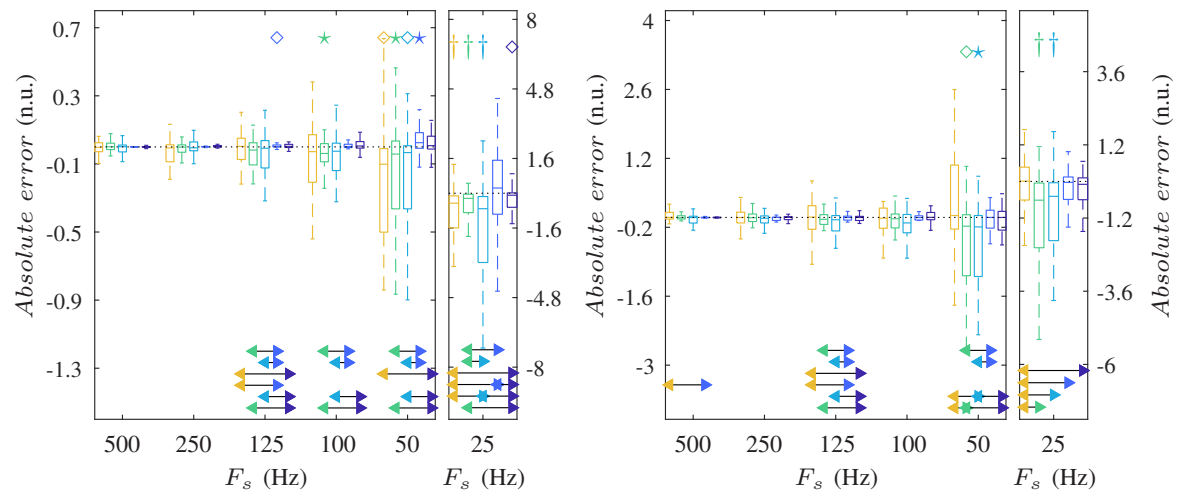

(d) $R_{\mathrm{LF} / \mathrm{HF}}$ : ratio between $\mathrm{LF}$ and HF power

Fig. 4: Estimated relative $\left(P_{\mathrm{LF}}, P_{\mathrm{HF}}\right)$ and absolute $\left(P_{\mathrm{LF}}, R_{\mathrm{LFFF}}\right)$ errors between frequency indices from PRV and PRV ${ }^{1000}$. The significance level $\alpha$ is indicated with $\star$ for $\alpha=0.05$, $\diamond$ for $\alpha=0.01$ and $\dagger$ for $\alpha=0.001$. Median of $e_{\mathrm{r}, \mathrm{PRV}, \mathrm{yi}_{\mathrm{i}}}^{f_{s}}>5 \%$ are indicated by a dot below the $\alpha$ level symbol. The arrow indicates statistically significant differences between fiducial points at the same rate using Friedman statistics with the Bonferroni multiple-comparison test. The colour of the start and end of the arrow indicates the fiducial point analysed. The dotted horizontal line represents the zero value in the error. The outliers are not plotted. 
TABLE I: Median (bias) and limits of agreement (LOA) values of the comparing paired RR and PP series. Results shown as bias, interquartile range (iqr) and [bias $+1.45 \cdot i q r$, bias $-1.45 \cdot i q r]$ values. All results are in seconds.

\begin{tabular}{|c|c|c|c|c|c|c|c|}
\hline & $f_{s}(\mathrm{~Hz})$ & & $n_{\mathrm{A}}$ & $n_{\mathrm{S}}$ & $n_{\mathrm{M}}$ & $n_{\mathrm{L}}$ & $n_{\mathrm{I}}$ \\
\hline \multirow{12}{*}{$\begin{array}{l}\overrightarrow{\mathbb{D}_{0}} \\
\overrightarrow{\mid \Xi}\end{array}$} & 1000 & $\begin{array}{l}\text { bias / iqr } \\
\text { LOA }\end{array}$ & $\begin{array}{c}0.001 / 0.069 \\
{[0.101,-0.099]}\end{array}$ & $\begin{array}{c}0.001 / 0.059 \\
{[0.087,-0.085]}\end{array}$ & $\begin{array}{c}0.001 / 0.059 \\
{[0.087,-0.085]}\end{array}$ & $\begin{array}{c}0.001 / 0.059 \\
{[0.087,-0.085]}\end{array}$ & $\begin{array}{c}0.001 / 0.059 \\
{[0.087,-0.085]}\end{array}$ \\
\hline & 500 & bias / iqr & $\begin{array}{c}0.002 / 0.069 \\
0.102-0.098]\end{array}$ & $0.001 / 0.058$ & $0.001 / 0.059$ & $0.001 / 0.059$ & $0.001 / 0.059$ \\
\hline & 250 & bias / iqr & $0.002 / 0.070$ & $0.001 / 0.058$ & $0.001 / 0.059$ & $0.001 / 0.060$ & $0.001 / 0.059$ \\
\hline & 250 & LOA & {$[0.103,-0.099]$} & {$[0.085,-0.083]$} & {$[0.087,-0.085]$} & {$[0.087,-0.086]$} & {$[0.087,-0.085]$} \\
\hline & 125 & bias / iqr & $0.002 / 0.069$ & $0.001 / c$ & $0.001 / 0.060$ & $0.001 / 0.060$ & $0.001 / 0.059$ \\
\hline & $12 J$ & LOA & {$[0.102,-0.098]$} & {$[0.085,-0.083]$} & {$[0.088,-0.086]$} & {$[0.087,-0.086]$} & {$[0.087,-0.085]$} \\
\hline & 10 & bias / iqr & $0.001 / 0.070$ & $0.001 / 0.059$ & $0.001 / 0.060$ & $0.001 / 0.060$ & $0.001 / 0.060$ \\
\hline & & LOA & {$[0.103,-0.101]$} & {$[0.087,-0.085]$} & {$[0.088,-0.086]$} & {$[0.088,-0.086]$} & {$[0.088,-0.086]$} \\
\hline & 50 & bias / iqr & $0.002 / 0.070$ & $0.001 / 0.061$ & $0.001 / 0.062$ & $0.001 / 0.060$ & $0.001 / 0.060$ \\
\hline & & LOA & {$[0.103,-0.099]$} & {$[0.089,-0.087]$} & {$[0.091,-0.089]$} & {$[0.088,-0.086]$} & {$[0.088,-0.086]$} \\
\hline & 25 & bias / iqr & $0.002 / 0.077$ & $0.002 / 0.067$ & $0.002 / 0.086$ & $0.001 / 0.093$ & $0.001 / 0.063$ \\
\hline & $2 J$ & LOA & {$[0.114,-0.110]$} & {$[0.099,-0.095]$} & {$[0.127,-0.123]$} & {$[0.135,-0.133]$} & {$[0.092,-0.090]$} \\
\hline \multirow{12}{*}{ 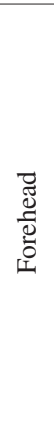 } & 1000 & $\begin{array}{l}\text { bias / iqr } \\
\text { LOA }\end{array}$ & $\begin{array}{c}0.002 / 0.090 \\
0.133,-0.129\end{array}$ & $\begin{array}{c}0.002 / 0.072 \\
{[0.106,-0.102]}\end{array}$ & $\begin{array}{c}0.002 / 0.074 \\
{[0.109,-0.105}\end{array}$ & $\begin{array}{c}0.002 / 0.074 \\
{[0.109,-0.105]}\end{array}$ & $\begin{array}{c}0.002 / 0.074 \\
{[0.109,-0.105]}\end{array}$ \\
\hline & & bias / iqr & $0.002 / 0.088$ & $0.002 / 0.072$ & $0.002 / 0.074$ & $0.002 / 0.074$ & $0.002 / 0.074$ \\
\hline & 50 & LOA & {$[0.130,-0.126]$} & {$[0.106,-0.102]$} & {$[0.109,-0.105]$} & {$[0.109,-0.105]$} & {$[0.109,-0.105]$} \\
\hline & 250 & bias / iqr & $0.002 / 0.090$ & $0.002 / 0.072$ & $0.002 / 0.074$ & $0.002 / 0.073$ & $0.002 / 0.073$ \\
\hline & 250 & LOA & {$[0.132,-0.128]$} & {$[0.106,-0.102]$} & {$[0.109,-0.105]$} & {$[0.109,-0.105]$} & {$[0.108$,} \\
\hline & 125 & bias / iqr & $0.002 / 0.090$ & $0.002 / 0.072$ & $0.002 / 0.072$ & $0.002 / 0.073$ & $0.002 / 0.073$ \\
\hline & & LOA & {$[0.133,-0.129]$} & {$[0.106,-0.102]$} & {$[0.106,-0.102]$} & {$[0.109,-0.104]$} & {$[0.108,-0.104]$} \\
\hline & 100 & bias / iqr & $0.002 / 0.090$ & $0.002 / 0.072$ & $0.002 / 0.073$ & $0.002 / 0.073$ & $0.002 / 0.072$ \\
\hline & & & {$[0.1$} & & 4] ] & 04] & 102] \\
\hline & 50 & bias / iqr & $0.002 / 0.090$ & $0.003 / 0.07$ & $0.002 / 0.074$ & $0.002 / 0.073$ & $0.002 / 0.073$ \\
\hline & & bias / iqr & $0.002 / 0.091$ & $0.002 / 0.077$ & $0.002 / 0.076$ & $0.002 / 0.074$ & $0.002 / 0.072$ \\
\hline & 25 & LOA & {$[0.134,-0.130]$} & {$[0.114,-0.110]$} & {$[0.112,-0.108]$} & {$[0.109,-0.105]$} & {$[0.106,-0.102]$} \\
\hline
\end{tabular}

to the fact that the interpolation at $1000 \mathrm{~Hz}$ is only sensitive to errors of at least $0.5 \mathrm{~ms}$, when the location of the fiducial point is estimated.

The bias and limits of agreement presented in Table I between RR and PP series exhibited close agreement between measures. The estimated bias was $0.002 \mathrm{~s}$ for all cases in the forehead-PPG signal, and $0.001 \mathrm{~s}$ in the finger-PPG signal, except for $n_{\mathrm{A}}$ and the lowest frequency of $n_{\mathrm{S}}$ and $n_{\mathrm{M}}$. The interquartile range was slightly increased when the sampling rate decreased, in the finger-PPG signal. The analysis showed a larger divergence in the forehead than in the finger measurements. More specifically, the limits of agreement (see Table I) in the forehead were higher, especially when $n_{\mathrm{A}}$ was used as fiducial point. In Fig. 1, it is observed that the forehead-PPG signal presents a smooth peak morphology, where the $n_{\mathrm{A}}$ is located. In this way, although its location can significantly change when the sampling rate drops, the change in PPG amplitude is usually much smaller. Thus $n_{\mathrm{M}}, n_{\mathrm{L}}$ and $n_{\mathrm{I}}$, remain more robust than $n_{\mathrm{A}}$, as they depend on the PPG amplitude at $n_{\mathrm{A}}$.

The HRV and PRV signals were obtained from the RR and PP series, respectively. Table II, Fig. 3 and Fig. 4, show the effect of a decrease in sampling rate in PRV indices. In general, the relative error obtained for the transmission-based-finger PPG signals are slightly lower than for the reflection-basedforehead PPG signals. The accuracy of the PRV estimation is highly dependent on the possible signal interferences or artefacts, and on the morphology of the PPG pulse, which depends on the signal-acquisition technique, and on the location of the sensor. The relative errors results shown in the Table II confirm that these errors were greater for $n_{\mathrm{A}}$ than for the other fiducial points. Previously, in Fig. 1, the smoother shapes of the reflection-based PPG waveforms were analysed and these results confirm that $n_{\mathrm{A}}$ was not the most suitable point for a PRV analysis due to its low robustness against noise, especially for forehead PPG signals. This observation is coherent with [18], where a sampling rate of $1000 \mathrm{~Hz}$ was used.

In agreement with other studies [1], our results showed higher relative errors between PRV and HRV for short-term variability indices $\overline{R M S S D}$ or $\overline{p N N 50}$ than for $\overline{P R M}$ and $\overline{S D N N}$. The variations in the $\overline{R M S D D}$ and $\overline{p N N 50}$ indices are known to reflect high frequency activity derived from parasympathetic activation [4]. Otherwise, variables that are known to reflect low frequency activity, as $\overline{P R M}$ and $\overline{S D N N}$, showed little or no significant changes. The biggest error occurs for $n_{\mathrm{A}}$ on the forehead PRV signal (Table II and Fig. 3). As previously mentioned, the location of $n_{\mathrm{A}}$ is greatly affected by the reduction in the sampling rate due to the smoother shapes of the PPG signal when recorded in the forehead. Moreover, low frequency indices showed lower relative errors than highfrequency indices between both variability signals [1], [33]. These differences are related to the fact that a slight variation of the fiducial point localization could increase the total power of the PRV signal; particularly HF power, which would be more affected since HF is related to short term changes on the time domain [15]. This increase in power is accentuated with the decrease in the sampling rate, as shown in Fig. 4 and Table II. As $P_{\mathrm{LF}}$ showed a smaller increase than $P_{\mathrm{HF}}$, the variables $R_{\mathrm{LF} / \mathrm{HF}}$ and $P_{\mathrm{LFn}}$ decreased, with more emphasis when the sampling rate decreases. This trend seems opposite in the case of the PRV forehead signal, when $n_{\mathrm{S}}, n_{\mathrm{M}}, n_{\mathrm{L}}$ and $n_{\mathrm{I}}$ were used as fiducial point, and the frequency was greater than $50 \mathrm{~Hz}$. In this case the increase in the $P_{\mathrm{HF}}$ power is much 
TABLE II: Estimated relative (time indices and $\left.P_{\mathrm{LF}}, P_{\mathrm{HF}}\right)$ and absolute $\left(P_{\mathrm{LF}}, R_{\mathrm{LFHF}}\right)$ errors between HRV and all PRV signals. Results shown as median/interquartile range values. The significance level $\alpha$ is indicated with $\star$ for $\alpha=0.05, \diamond$ for $\alpha=0.01$ and $\dagger$ for $\alpha=0.001$. The row $H R V$ shows the reference values extracted from the HRV signal.

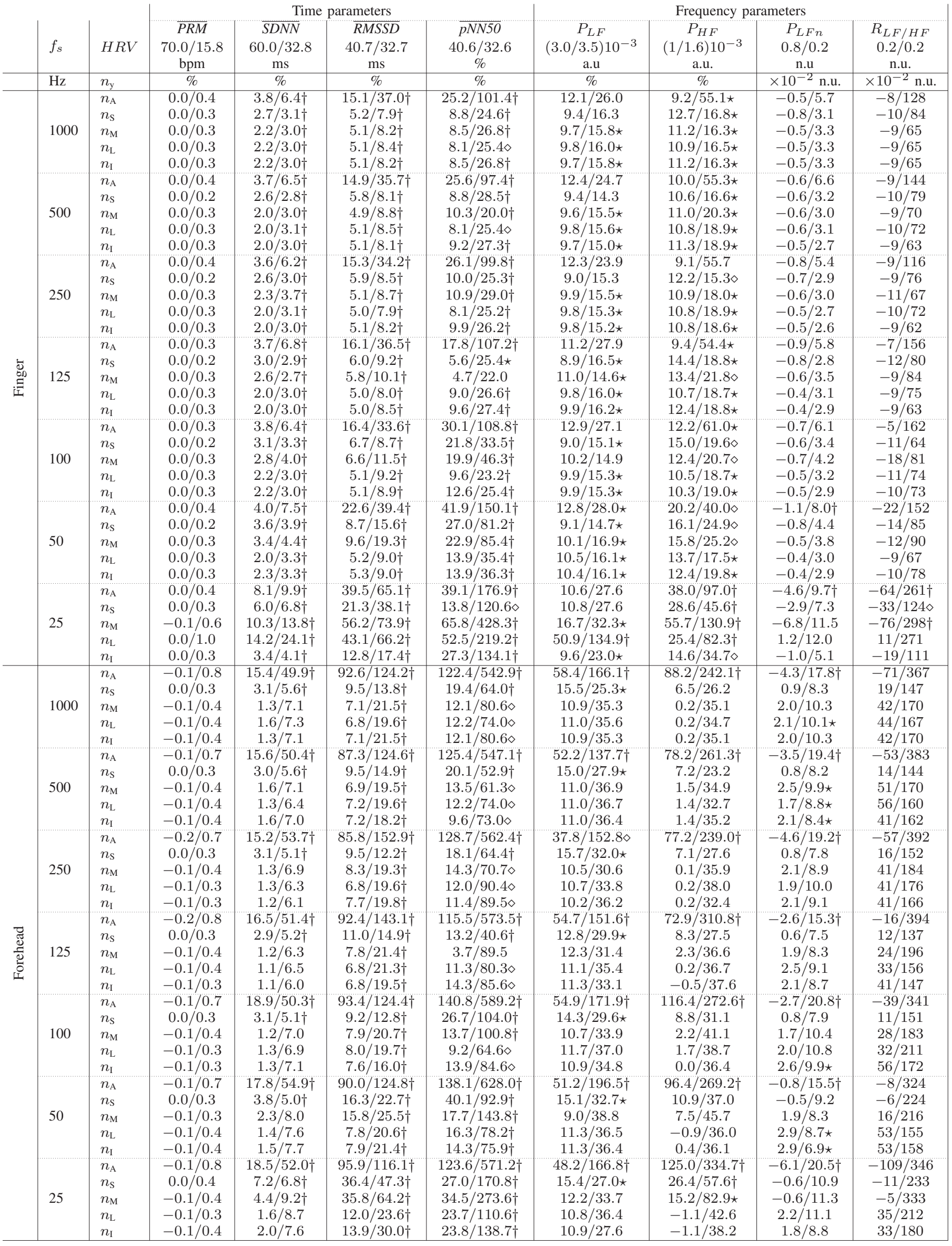


lower than the one observed in $P_{\mathrm{LF}}$ (Table II).

The changes observed in Table II are not only due to the reduction of the sampling rate, but also to the differences between the HRV and PRV signals. To analyse the error due only to the reduction of the sampling frequency, a error was calculated using $\mathrm{PRV}^{1000}$ as reference. This error is shown in Fig. 3 and Fig. 4. These figures show that the relative errors in the time and frequency indices between PRV and $\mathrm{PRV}^{1000}\left(e_{\mathrm{r}, \mathrm{PRV}, \mathrm{yi}}^{f_{S}}\right)$ increase with the decrease in the sampling rate. However, this error is always less when $n_{\mathrm{L}}$ or $n_{\mathrm{I}}$ was used as fiducial point, both for the signal recorded on the finger and on the forehead. Note that in the case of finger-PPG, for the lower frequency, the $n_{\mathrm{L}}$ point shows a greater error than the $n_{\mathrm{I}}$, which seems to indicate that $n_{\mathrm{L}}$ is more sensitive to the propagation of the error performed in the estimation of $n_{\mathrm{A}}$ and $n_{\mathrm{B}}$ points. In these figures, we can also see that the median relative error only exceeds $5 \%$ for sampling rate below $100 \mathrm{~Hz}$, for most fiducial points. For the $n_{\mathrm{I}}$, the median of the $e_{\mathrm{r}, \mathrm{PRV}, \mathrm{yi}}^{f_{s}}$ only exceeds $5 \%$ in $\overline{R M S D D}$ and $\overline{p N N 50}$, when the frequency is reduced to $25 \mathrm{~Hz}$ (Fig. 3.c and .d).

In the case of $P_{\mathrm{LF}}$ error, it only exceeds $5 \%$ for $n_{\mathrm{L}}$ at $25 \mathrm{~Hz}$ in finger PRV signal (Fig. 4.a). The $P_{\mathrm{HF}}$ error was always below $5 \%$, when $n_{\mathrm{I}}$ was used as fiducial point (Fig. 4.b). For the rest of fiducial points, the $5 \%$ error was only exceeded for $25 \mathrm{~Hz}$, except in the case of $n_{\mathrm{M}}$ which occurs at $50 \mathrm{~Hz}$ for forehead PRV signal. In this case, for $n_{\mathrm{S}}$ and $n_{\mathrm{M}}$ in the forehead PRV signal, it can be seen that the $P_{\mathrm{HF}}$ power increases with the decrease in the sampling rate (Fig. 4.b), causing a reduction in $P_{\mathrm{LFn}}$ and $R_{\mathrm{LF} / \mathrm{HF}}$ indices (Fig. 4.c and .d). For $n_{\mathrm{L}}$ and $n_{\mathrm{I}}$, the same trend was observed for a sampling rate of $25 \mathrm{~Hz}$, but not for higher frequencies since the error was lower and no trend could be observed.

Finally, the results of the Friedman test among the five fiducial points showed significant differences in most of the studied indices for sampling rates from $125 \mathrm{~Hz}$ and below, so for these sampling rates the best results would be obtained when using $n_{\mathrm{I}}$ as fiducial point.

Other authors demonstrated that the medium fiducial and up-slope points are more suitable for PRV analysis, when the sampling rate was $1000 \mathrm{~Hz}$ [18]. However, reducing the sampling rate of the PPG signal introduces an error in the location of this point that can be corrected by interpolating the ascending pulse region at $1000 \mathrm{~Hz}$, in the case of medium point. Based on our results, the medium interpolate and the line-medium points of the PPG are proposed as the most accurate in different PPG morphologies and sensor locations. The results in Fig. 2 show that the absolute error was 0 for $n_{\mathrm{I}}$ if the sampling rate drops down to $100 \mathrm{~Hz}$ for infrared wavelength (125 Hz for red wavelength), when the signal is recorded on the finger, and to $250 \mathrm{~Hz}$ (the same for the red wavelength) when it is recorded on the forehead. In the case of $n_{\mathrm{L}}$, these errors are below $0.5 \mathrm{~ms}$.

Fig. 3 and Fig. 4 show that the relative error was practically zero for sampling rate above $50 \mathrm{~Hz}$, when $n_{\mathrm{L}}$ or $n_{\mathrm{I}}$ were considered as fiducial point, and significant differences in time and frequency indices appear when PRV ${ }^{1000}$ was used as a reference, for sampling rates below. However, for these fiducial points, the median of the $e_{\mathrm{r}, \mathrm{PRV}}^{f_{s}}$ does not exceed
$5 \%$ for sampling rate above $25 \mathrm{~Hz}$. Based on these results, the sampling rate of the PPG signal recorded on the finger and forehead could be lowered to $50 \mathrm{~Hz}$, without causing significant changes in the time and frequency indices, if $n_{\mathrm{L}}$ or $n_{\mathrm{I}}$ are used as fiducial point. Selecting a lower sampling rate will depend on whether the error performed in the estimation of time and frequency indices is less than the expected change of these indices in each specific application.

The main aim of this work is to identify the impact of the PPG sampling rate in the PRV indices. Thus, the studied data is primarily composed of signals from young healthy individuals, recorded during stationary conditions. In future studies, nonstationary conditions will be considered, exposing the subjects to situations that modify their ANS response, such as stress states, or postural changes (tilt test).

\section{CONCLUSION}

The widespread use of watches and smart-bands with integrated PPG sensors provides a new field for the development of applications based on PRV analysis. The resources are very limited in these applications, making very interesting to reduce the sampling rate of the PPG signal as much as possible while maintaining a desired level of robustness. In this work we have studied how this reduction in sampling rate can affect to the measures of PRV indices, extracted from four PPG signals: PPG recorded in finger or forehead and with red or infrared wavelength. Five different fiducial points have been considered: apex, up-slope, medium line-medium and medium interpolate points. The results showed that the best performance was obtained when line-medium or medium interpolate point were used as fiducial points. These points allowed a reduction of the sampling rate of the PPG signal, recorded in the finger and forehead, to $50 \mathrm{~Hz}$ without causing significant changes in PRV indices with respect to those obtained when using a sampling rate of $1000 \mathrm{~Hz}$. Likewise, it is not recommended to use, in any case, the apex point when the PPG waveform is smooth, as when it is recorded on the forehead. On the finger, the apex, up-slope and medium point are not recommended to use them below $250 \mathrm{~Hz}$, unless the expected change is greater than the error that occurs.

\section{ACKNOWLEDGEMENTS}

This work has received funding from Ministerio de Ciencia, Innovación y Universidades, from FEDER through the projects PGC2018-095936-B-I00 and RTI2018-097723-B-I00, from Centro Universitario de la Defensa (CUD) under the projects UZCUD2019-TEC-01 and CUD2019-10, from Aragón Government and European Regional Development Fund through Grupo de Referencia BSICoS (Biomedical Signal Interpretation \& Computational Simulation, T39-17R), and from the European Unions Framework Programme for Research and Innovation Horizon 2020 (2014-2020) under the Marie SkodowskaCurie Grant Agreement No. 745755. The computation was performed by the ICTS NANBIOSIS, specifically by the High Performance Computing Unit of CIBER-BBN at University of Zaragoza. This work would never have been done without the collaboration of the Hospital General de la Defensa en 
Zaragoza, and the assistance as volunteers of the Regimiento de Pontoneros y Especialidades de Ingenieros $n^{o} 12$.

\section{REFERENCES}

[1] A. Schäfer and J. Vagedes, "How accurate is pulse rate variability as an estimate of heart rate variability?: A review on studies comparing photoplethysmographic technology with an electrocardiogram," pp. 1529, 2013.

[2] S. Lu, H. Zhao, K. Ju, K. Shin, M. Lee, K. Shelley, and K. H. Chon, "Can photoplethysmography variability serve as an alternative approach to obtain heart rate variability information?" Journal of Clinical Monitoring and Computing, vol. 22, no. 1, pp. 23-29, 2008.

[3] N. Selvaraj, A. Jaryal, J. Santhosh, K. K. Deepak, and S. Anand, "Assessment of heart rate variability derived from finger-tip photoplethysmography as compared to electrocardiography," Journal of Medical Engineering and Technology, vol. 32, no. 6, pp. 479-484, 2008.

[4] Task Force of the European Society of Cardiology the North American Society of Pacing and Electrophysiology, "Heart rate variability standards of measurement, physiological interpretation, and clinical use," Circulation, vol. 93, no. 5, pp. 1043-1065, 1996.

[5] M. Peláez, M. T. Lozano, A. Hernando, M. Aiger, and E. Gil, "Photoplethysmographic waveform versus heart rate variability to identify lowstress states: Attention test," IEEE Journal of Biomedical and Health Informatics, vol. 23, no. 5, pp. 1940-1951, Sep. 2019.

[6] A. Hernando, M. Peláez, M. T. Lozano, M. Aiger, D. Izquierdo, A. Sanchez, M. I. Lopez-Jurado, J. I. Moura, J. Fidalgo, J. Lázaro, and E. Gil, "Autonomic nervous system measurement in hyperbaric environments using ECG and PPG signals," IEEE Journal of Biomedical and Health Informatics, vol. 23, no. 1, pp. 132-142, Jan 2019.

[7] J. Lázaro, E. Gil, J. M. Vergara, and P. Laguna, "Pulse rate variability analysis for discrimination of sleep-apnea-related decreases in the amplitude fluctuations of pulse photoplethysmographic signal in children," IEEE Journal of Biomedical and Health Informatics, vol. 18, no. 1, pp. 240-246, 2014.

[8] J. Yao, X. Sun, and Y. Wan, "A pilot study on using derivatives of photoplethysmographic signals as a biometric identifier," in Annual International Conference of the IEEE Engineering in Medicine and Biology - Proceedings, 2007, pp. 4576-4579.

[9] A. Reisner, P. A. Shaltis, D. McCombie, and H. H. Asada, "Utility of the photoplethysmogram in circulatory monitoring," pp. 950-958, 2008.

[10] V. Vizbara, A. Solosenko, D. Stankevicius, and V. Marozas, "Comparison of green, blue and infrared light in wrist and forehead photoplethysmography," in Biomedical Engineering 2014, 2013, pp. 78-81. [Online]. Available: http://biomed.ktu.lt/index.php/BME/article/view/460

[11] Y. Maeda, M. Sekine, and T. Tamura, "The advantages of wearable green reflected photoplethysmography," in Journal of Medical Systems, vol. 35, no. 5, 2011, pp. 829-834.

[12] AHA-Committee, "Recommendations for standardizations of leads and specifications for instruments in ecg and vcg," Circulation, vol. 52, JulyDec. 1975.

[13] P. Schluter, D. Clapham, J. Mertens, A. Mockaitis, D. Mortara, and K. Stankus, "Design and evaluation of a computer based system for holter tape analysis," in Computers in Cardiology, 1984, pp. 193-196.

[14] M. Merri, D. C. Farden, J. G. Mottley, and E. L. Titlebaum, "Sampling Frequency of the Electrocardiogram for Spectral Analysis of the Heart Rate Variability," IEEE Transactions on Biomedical Engineering, vol. 37, no. 1, pp. 99-106, 1990.

[18] E. Peralta, J. Lazaro, R. Bailon, V. Marozas, and E. Gil, "Optimal fiducial points for pulse rate variability analysis from forehead and finger photoplethysmographic signals," Physiological Measurement, vol. 40, no. 2, p. 025007, feb 2019. [Online]. Available: https://doi.org/10.1088/1361-6579/ab009b
[15] A. Choi and H. Shin, "Photoplethysmography sampling frequency: Pilot assessment of how low can we go to analyze pulse rate variability with reliability?" Physiological Measurement, vol. 38, no. 3, pp. 586-600, 2017.

[16] N. D. Giardino, P. M. Lehrer, and R. Edelberg, "Comparison of finger plethysmograph to ECG in the measurement of heart rate variability," Psychophysiology, vol. 39, no. 2, pp. 246-253, 2002.

[17] S. Béres, L. Holczer, and L. Hejjel, "On the Minimal Adequate Sampling Frequency of the Photoplethysmogram for Pulse Rate Monitoring and Heart Rate Variability Analysis in Mobile and Wearable Technology," Measurement Science Review, vol. 19, no. 5, pp. 232-240, 2019.

[19] S. Rajala, H. Lindholm, and T. Taipalus, "Comparison of photoplethysmogram measured from wrist and finger and the effect of measurement location on pulse arrival time," Physiological Measurement, vol. 39, no. 7,2018 .

[20] M. C. Hemon and J. P. Phillips, "Comparison of foot finding methods for deriving instantaneous pulse rates from photoplethysmographic signals," Journal of Clinical Monitoring and Computing, vol. 30, no. 2, pp. 157168,2016

[21] D. Buxi, J. M. Redouté, and M. R. Yuce, "A survey on signals and systems in ambulatory blood pressure monitoring using pulse transit time," Physiological Measurement, vol. 36, no. 3, pp. R1-R26, 2015.

[22] D. Sokas, M. Gailius, and V. Marozas, "Diver physiology monitor and its graphical user interface," in Proceedings of International Scientific - Practical Conference, Virtual Instruments in Biomedicine, 2016, pp. $5-9$.

[23] L. Sörnmo and P. Laguna, Bioelectrical Signal Processing in Cardiac and Neurological Applications, 2005.

[24] J. P. Martinez, R. Almeida, S. Olmos, A. Rocha, and P. Laguna, "A wavelet-based ECG delineator: evaluation on standard databases," IEEE Transactions on Biomedical Engineering, vol. 51, no. 4, pp. 570-581, April 2004

[25] J. Mateo and P. Laguna, "Analysis of heart rate variability in the presence of ectopic beats using the heart timing signal," IEEE Transactions on Biomedical Engineering, vol. 50(3), pp. 334-343, 2003.

[26] J. Lázaro, E. Gil, R. Bailón, A. Mincholé, and P. Laguna, "Deriving respiration from photoplethysmographic pulse width," Medical and Biological Engineering and Computing, vol. 51, no. 1-2, pp. 233-242, 2013.

[27] E. Gil, J. M. Vergara, and P. Laguna, "Detection of decreases in the amplitude fluctuation of pulse photoplethysmography signal as indication of obstructive sleep apnea syndrome in children," Biomedical Signal Processing and Control, vol. 3, no. 3, pp. 267-277, 2008.

[28] R. Bailón, G. Laouini, C. Grao, M. Orini, P. Laguna, and O. Meste, "The integral pulse frequency modulation model with time-varying threshold: Application to heart rate variability analysis during exercise stress testing," Biomedical Engineering, IEEE Transactions on, vol. 58, no. 3, pp. 642-652, March 2011.

[29] J. Martin Bland and D. G. Altman, "Statistical methods for assessing agreement between two methods of clinical measurement," The Lancet, vol. 327 , no. 8476, pp. 307-310, 1986.

[30] R. Peck, C. Olsen, and J. L. Devore, Introduction to Statistics and Data Analysis, 5th ed. Brooks Cole, 2015.

[31] Y. Mendelson and B. D. Ochs, "Noninvasive Pulse Oximetry Utilizing Skin Reflectance Photoplethysmography," IEEE Transactions on Biomedical Engineering, vol. 35, no. 10, pp. 798-805, 1988.

[32] B. A. Fallow, T. Tarumi, and H. Tanaka, "Influence of skin type and wavelength on light wave reflectance," Journal of Clinical Monitoring and Computing, vol. 27, no. 3, pp. 313-317, 2013.

[33] E. Gil, M. Orini, R. Bailón, J. M. Vergara, L. Mainardi, and P. Laguna, "Photoplethysmography pulse rate variability as a surrogate measurement of heart rate variability during non-stationary conditions," Physiological Measurement, vol. 31, no. 9, pp. 1271-1290, 2010. 